[0212-7199 (2007) 24: 6; pp 281-284] ANALES DE MEDICINA INTERNA Copyright $(C) 2007$ ARAN EDICIONES, S.L.

AN. MED. INTERNA (Madrid) Vol. 24, N. ${ }^{\circ}$ 6, pp. 281-284, 2007

\title{
Nuevas posibilidades diagnósticas y terapéuticas en el síndrome de Mirizzi
}

\author{
J. SÁNCHEZ BEORLEGUI, R. CABEZALI SÁNCHEZ, E. MONSALVE LAGUNA, \\ P. SORIANO GIL ALBARELLOS, N. MORENO DE MARCOS
}

Servicio de Cirugía General y Aparato Digestivo. Fundación Hospital de Calahorra. Calahorra. La Rioja

\author{
NEW DIAGNOSTIC AND SURGICAL APPROACH TO MIRIZZI SYN- \\ DROME
}

\begin{abstract}
RESUMEN
El síndrome de Mirizzi es una variante poco frecuente de colelitiasis en la que un cálculo impactado en la bolsa de Hartmann comprime la vía biliar desencadenando una ictericia obstructiva, frecuentemente seguida de fenómenos inflamatorios y de diversas complicaciones (colecistitis, colangitis, fístulas etc).

Presentamos dos pacientes con síndrome de Mirizzi, correctamente diagnosticados en el preoperatorio e intervenidos por vía laparoscópica. Un caso fue convertido por adherencias en el triángulo de Calot y tratado con colecistectomía subtotal y coledocorrafia sobre tubo en T de Kher. En el otro enfermo se pudo completar con éxito el procedimiento. Ambos postoperatorios cursaron con normalidad.

En el presente artículo, se analizan las técnicas diagnósticas que ayudan a una identificación precoz del síndrome y se discuten las opciones terapéuticas más adecuadas en el momento actual, prestando una especial atención al papel de la colangiopancreatografía endoscópica y del abordaje laparoscópico en el manejo de estos pacientes.
\end{abstract}

PALABRAS CLAVE: Colelitiasis. Obstrucción de la vía biliar. Síndrome de Mirizzi. Diagnóstico. Tratamiento endoscópico. Cirugía Laparoscópica.

\begin{abstract}
Mirizzi's syndrome is an unusual complication of gallstone disease, in which a stone impacting in the neck of the gallbladder (Hartmann's pouch) compresses the common bile duct. This mechanical obstruction leads to obstructive jaundice frequently followed by inflammatory changes and several complications.

We present two patients affected by Mirizzi's syndrome whose diagnosis was correct in the preoperative period and approached by laparoscopy. A case was converted to open procedure due to adhesions in the Calot's triangle, and therefore, treated with subtotal cholecystectomy and choledochorrhaphy over a T tube. In the other case the laparoscopy access became successful. Both postoperative courses were uneventful.

In this article, suitable diagnostic techniques are analyzed. On the other hand, we discuss what is the best therapeutic option, with especial attention to the relevance of endoscopic retrograde cholangiopancreatography and laparoscopic approach in the management of those patients.
\end{abstract}

KEY WORDS: Gallstone disease. Bile duct obstruction. Mirizzi syndrome. Diagnostic. Endoscopic treatment. Laparoscopic surgery.

Sánchez Beorlequi J, Cabezali Sánchez R, Monsalve Laguna E, Soriano Gil-Albarellos P, Moreno de Marcos N. Nuevas posibilidades diagnósticas y terapéuticas en el síndrome de Mirizzi. An Med Interna (Madrid) 2007; 24: 281-284.

\section{INTRODUCCIÓN}

El epónimo síndrome de Mirizzi (SM) define un complejo anatomo-clínico característico dentro de la patología quirúrgica biliar. La secuencia evolutiva se inicia con una colelitiasis y la impactación de un lito en la bolsa de Hartmann o en el conducto cístico, dando origen a una colostasis por compresión extrínseca de la vía biliar principal (VBP) $(1,2)$. A partir de ese momento, pueden desencadenarse diversos fenómenos como ictericia, exclusión vesicular, colecistitis aguda, fístula colecisto-coledociana o bilio-digestiva, coledocolitiasis, colan- gitis y cirrosis biliar secundaria, entre otros. La secuencia de estos signos y síntomas no siempre es lógica y ordenada, según la gravedad de cada complicación. De forma característica el cuadro suele ser silente y se presenta como una ictericia intermitente, un hallazgo inesperado en el transcurso de una colecistectomía laparoscópica (CL) o debuta en fases avanzadas, con un síndrome de Bouveret (1-3).

Desde que fue descrito por Pablo Mirizzi en 1948, el enfoque terapéutico se basaba en la cirugía abierta. En aquellos años, los medios diagnósticos dificultaban una identificación preoperatoria $\mathrm{y}$, por tanto, la posibilidad de planificar una

Trabajo aceptado: 16 de enero de 2007 
estrategia distinta a la solución de la ictericia obstructiva en un quirófano. Por este motivo, la clasificación propuesta por Mirizzi o las de Mc Sherry o Csendes, nunca cumplieron la intención con que fueron diseñadas: correlacionar los hallazgos clínicos, anatómicos y exploratorios con un tratamiento quirúrgico adecuado $\mathrm{y}$, ante todo, precoz.

Hoy día, las técnicas diagnósticas y terapéuticas permiten diagnosticar y tratar el SM en fases tempranas, aumentando las posibilidades de curación, especialmente, cuando se trata de ancianos (1). La colangiorresonancia magnética (CRM), el TAC helicoidal con colangiograma, la colangiopancreatografía endoscópica (CPRE), las endoprótesis biliares o la vía laparoscópica son algunas novedades que permiten abordar el SM bajo nuevas prespectivas $(4,5)$.

En el presente artículo presentamos dos casos de SM e intentaremos responder a los interrogantes que, en nuestra opinión, se le plantean al clínico y al cirujano en la actualidad: a) Valor de las pruebas de imagen en el diagnóstico temprano del SM, antes de que se complique; b) Posibilidades de la CPRE, como tratamiento definitivo o previa a la cirugía; y c) Indicaciones y técnica de la CL.

\section{CASOS APORTADOS}

-Caso 1: Varón de 80 años con cuadro de 10 días de malestar general, ictericia progresiva, prurito, vómitos y dolor en hipocondrio derecho. En la ecografía apareció una colelitiasis múltiple, pared vesicular engrosada, un gran lito enclavado en el Hartmann, radicales izquierdos dilatados y colédoco de calibre normal, sin descartar coledocolitiasis. En la analítica se apreciaba un discreto movimiento de transaminasas e hiperbilirrubinemia, en sus fracciones total 4,63 mg/dl y directa $3,97 \mathrm{mg} / \mathrm{dl}$. El resto de cifras de la fórmula, coagulación y bioquímica eran normales, salvo leucocitosis $<15$ x 103/ul e insuficiencia renal leve de origen pre-renal. El paciente ingresa, instaurándose tratamiento médico, con evolución favorable, y es dado de alta al octavo día. Es citado en consulta para estudio, solicitándose un TAC abdominal con colangiograma. Dado que el resultado no resulta concluyente, se cursa una petición de CRM (Fig. 1) y, ante los hallazgos, una CPRE preferente (Fig. 1); sentándose la indicación operatoria bajo el diagnóstico de SM. La intervención se inicia por vía laparoscópica, con posterior conversión a cirugía abierta. Se practica una colecistectomía parcial y coledocorrafia sobre tubo en $\mathrm{T}$ de $\mathrm{Kehr}$ (Fig. 2). El postoperatorio cursa sin complicaciones y el paciente es dado de alta al sexto día, con su tubo en " $T$ " pinzado, que se retira a las 3 semanas tras una colangiografía normal.

-Caso 2: Varón de 83 años, con antecedentes de cólicos biliares de repetición y colelitiasis, fibrilación auricular tratada con dicumarínicos, inótropos y diuréticos, además de una EPOC estable. Ingresa en Medicina Interna por cuadro de ictericia indolora de 48 horas de evolución, sin fiebre ni intolerancia. Los valores de bilirrubina total eran de $6,83 \mathrm{mg} / \mathrm{dl}$ y directa $4,97 \mathrm{mg} / \mathrm{dl}$, con un INR de 2,72 y tiempo de protrombina de $38 \%$. La fórmula, bioquímica y enzimas hepáticas no estaban alteradas significativamente. En la ecografía se apreciaba un gran cálculo infundibular de unos $2,5 \mathrm{~cm}$ de diámetro, con distensión y edema mural, que improntaba en la VBP, causando una moderada dilatación del hepático y del árbol biliar. El diagnóstico fue de SM. Se procede a la suspensión de los dicumarínicos que son sustituidos por heparina de bajo peso molecular subcutánea. A las 48 horas se practica una CL urgente diferida, procedimiento que se realiza sin incidencias ni dificultades técnicas reseñables. A las 48 horas el enfermo es dado de alta para control ambulatorio, con la ictericia en franca resolución y valores casi normalizados de bilirrubina.
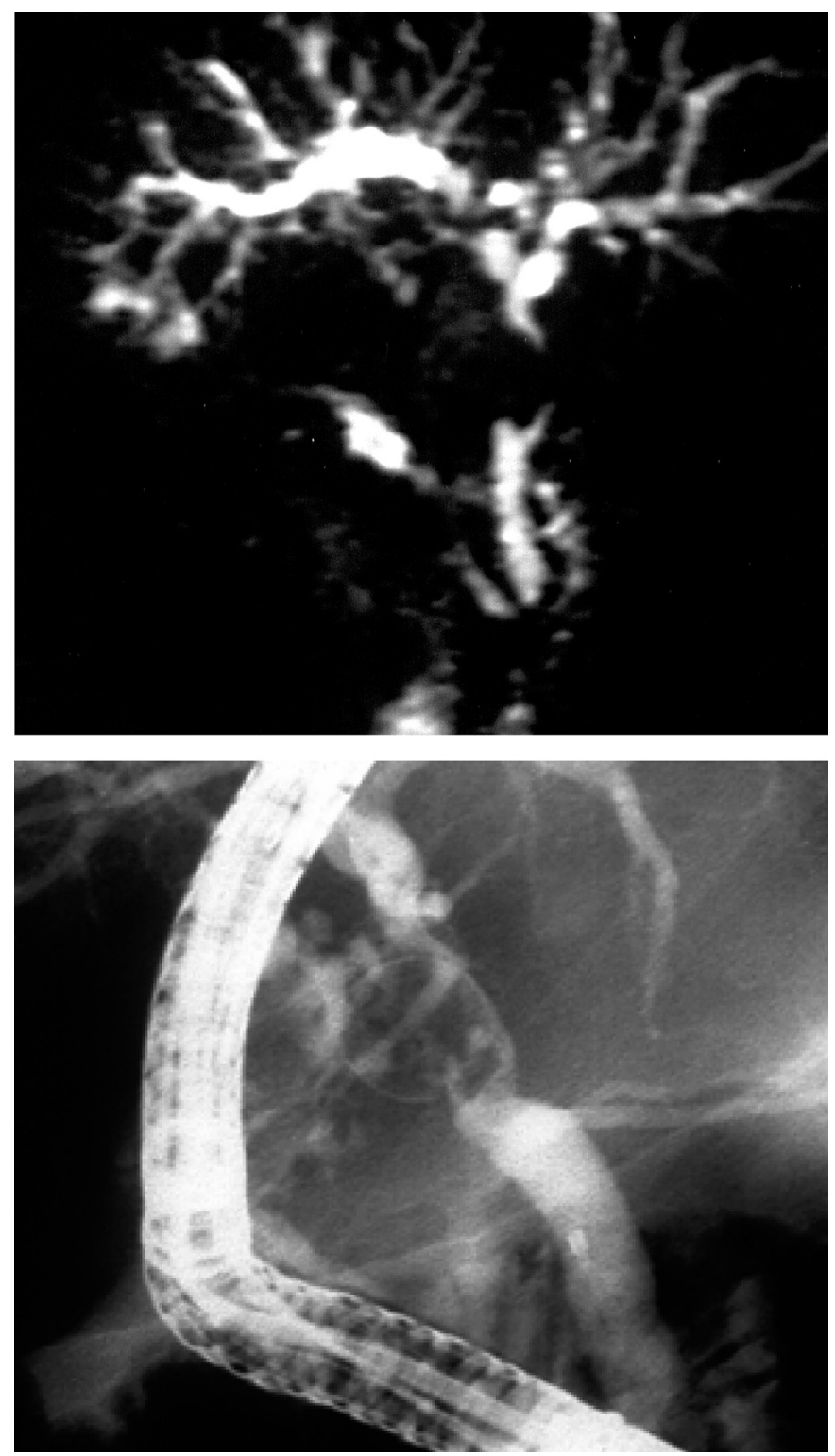

Fig. 1. Pruebas de imagen en el caso 1. De arriba a abajo: Colangiorresonancia magnética en la que aparece una imagen compatible con cálculo de unos $2 \mathrm{~cm}$ de diámetro en la confluencia hepatocística que condiciona la dilatación de la vía biliar intrahepática. CPRE. anulación selectiva de la VBP observándose una estenosis del colédoco proximal por la impronta de un cálculo de unos 25 $\mathrm{mm}$ de diámetro enclavado en el conducto cístico (Mirizzi tipo I). Se realiza esfinterotomía endoscópica amplia sin complicaciones, apreciándose la salida de material purulento.

\section{DISCUSIÓN}

La frecuencia con que se presenta el síndrome de Mirizzi en las series de colelitiasis es muy variable: inferior al 0,5\% en el primer mundo y en torno a un 4-5\% en los países en vías de desarrollo (6); siendo un 4,3\% en nuestra casuística de CL en octogenarios 2002-06 ( 2 casos en 46 intervenciones). Esta disparidad de cifras se relaciona con los medios diagnósticos disponibles y la prontitud con la que se trata la patología quirúrgica de la vía biliar en cada sistema sanitario. En las fases tempranas del SM, ante un paciente con cólico persistente o 


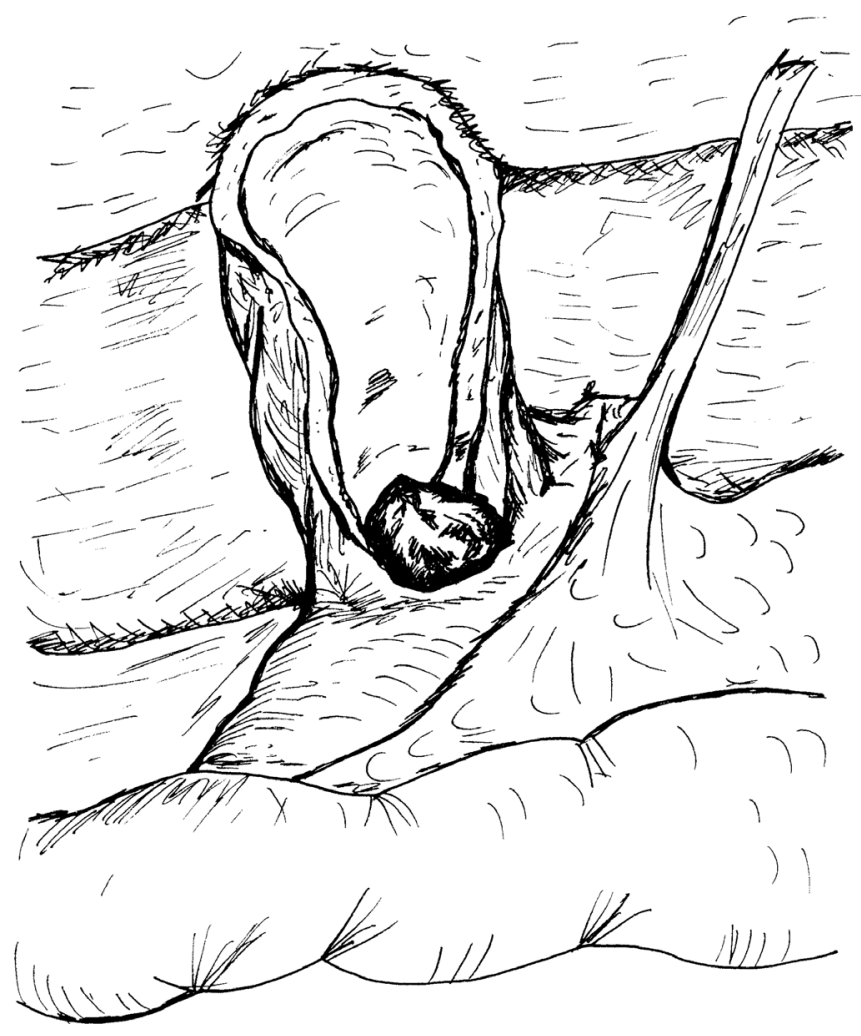

Fig. 2. Hallazgos operatorios en el Caso 1. Tras abordaje laparoscópico e intento infructuoso de disección del triángulo de Calot por las intensas adherencias inflamatorias en el triángulo de Calot, conversión a laparotomía subcostal derecha. Se observa un vesícula excluida, de pared engrosada y con microlitiasis. Se practica una colecistectomía parcial, preservando tejidos de la bolsa de Hartmann. Un grueso lito de $2 \mathrm{~cm}$ de diámetro, que se extrae, asoma entre las intensas adherencias inflamatorias que dificultan correcta identificación de la VBP. Se realiza una colangiografía intraoperatoria en la que se evidencia la fusión del cístico distal con el colédoco formando una fístula amplia (Mirizzi tipo II) y un buen paso de contraste al duodeno. La intervención concluye con una coledocoplastia sobre tubo en " $T$ " de Kher, utilizando los restos vesiculares y la colocación de un drenaje de Jackson-Pratt ambiental.

ictericia, una ecografía podrá aportar datos relevantes que lo sugieran. Los equipos de última generación y un radiólogo experto pueden ofrecer excelentes prestaciones sin necesidad de recurrir a otras pruebas más complejas y no siempre disponibles. Si existen dudas, con una TAC helicoidal o, más específicamente, una CRM, podremos conseguir un diagnóstico definitivo de SM, además de descartar un carcinoma de vesícula. Con estos datos planificaremos la estrategia terapéutica en función del estado del paciente y nuestras disponibilidades. Por desgracia, en la mayoría de los casos, la sobreinfección va a ser la norma y, como consecuencia de la sinergia entre los procesos inflamatorios, la flogosis y la isquemia, aparecerá la fístula colecisto-coledociana, coledocolitiasis, ictericia obstructiva y colangitis. Cuando esto sucede ya no puede demorarse el tratamiento por más tiempo. Si disponemos de un equipo entrenado en CPRE es posible plantear el abordaje endoscópico de la VBP, la remoción de los cálculos y drenar la bilis infectada. En enfermos sépticos, graves, descompensados o que no son subsidiarios de un segundo tiempo quirúrgico la CPRE puede resultar diagnóstica y terapéutica, curando al paciente (5). La colación de una endoprótesis en la VBP previene la compresión extrínseca y la aparición de nuevos episodios de ictericia, aunque no esta exenta de complicaciones $(7,8)$.

El tratamiento operatorio del SM es un tema de controversia permanente según se han incorporado nuevos avances en el desempeño de la cirugía (9-11). Una secuencia lógica podría ser la siguiente.

1. Si no se dispone de experiencia, equipo entrenado o el aparataje adecuado realizaremos un abordaje por laparotomía $(12,13)$.

2. Es preferible la vía abierta si se conoce de antemano la presencia de una fístula colecisto-coledociana, una coledocolitiasis o se presupone una intensa inflamación local (absceso subhepático, colecistitis gangrenosa, etc.) Las técnicas laparoscópicas para la exploración instrumental de la VBP no están al alcance de todos los cirujanos; pero también pueden estar contraindicadas, pues el estado del enfermo reclama una cirugía resolutiva y desaconseja una anestesia de varias horas o un neumoperitoneo prolongado $(8,14)$.

3. En muchos casos la CPRE preoperatoria puede ser decisiva para facilitar el uso de la vía laparoscópica. Por ejemplo, si ha descartado la presencia de fístulas biliares complejas, conseguido la remoción de la coledocolitiasis y la evacuación de la bilis purulenta.

4. Una postura lógica, sería intentar el abordaje laparoscópico, salvo contraindicación, y valorar según los hallazgos las opciones quirúrgicas en función de nuestro nivel técnico y recursos materiales (8). En nuestra opinión, un cirujano siempre debe tener presente que la laparoscopia es una simple vía de abordaje y no un fin en sí misma (15).

Bien sea por una u otra vía los objetivos operatorios son los mismos, y no han cambiado desde que fueron definidos por Mirizzi:

- Identificar el síndrome, especialmente cuando no se sospecha, por carecer de un diagnóstico preoperatorio $(1,4,8,16)$.

- Realizar una colecistectomía económica, preservando tejidos que pueden resultar vitales para la reparación del hepatocolédoco $(5,17,18)$.

-Exploración sistemática de la VBP, identificando las estructuras biliares mediante coledocoscopia o calangiografía intraoperatoria. De esta manera, prevenimos lesiones yatrógenas de difícil reparación $(2,4,12)$.

-Drenaje de la vía biliar, reconstruyendo la fístula con tejidos vesiculares sobre un tubo en " $\mathrm{T}$ " o con endoprótesis (8). Si la magnitud de las lesiones en la VBP hace inviable este proceder, se aplicaran procedimientos más complejos como una coledoco-duodenostomía o una hepático-yeyunostomía en Y de Roux (1).

Los casos presentados resumen muy bien la evolución clínica del SM y el enfoque diagnóstico y terapéutico vigente en la actualidad. Sin duda, el diagnóstico precoz en el paciente con colostasis e ictericia es la clave que permitirá la descompresión inmediata de la VBP mediante una CL urgente o urgente diferida $(15,19,20)$. Sin embargo, en enfermos complicados con colecistitis, colangitis y deterioro del estado general, es probable que se haya formado una fístula biliar y una coledocolitiasis. En estos pacientes, la CPRE esta altamente indicada, bien sea como paso previo a la cirugía o como un tratamiento definitivo. La elección de la vía de abordaje operatorio es un tema de debate sin resolver: algunos autores inciden en el aumento de la morbilidad y complicacio- 
nes de la vía laparoscópica mientras que para otros es electiva, excepto en casos extremos $(2,4,13)$. La tasa de conversión acumulada en la Literatura es de un $32 \%$, por lo que pasar a la vía abierta es una opción válida, prudente y que puede salvar la vida del enfermo (12).

Como conclusión, el SM es un cuadro complejo, dinámico y evolutivo. A partir del momento en que se produce la impactación del cálculo se desencadenarán, de forma inexorable la ictericia obstructiva, los fenómenos inflamatorios, la colangitis y la destrucción progresiva de la anatomía del árbol biliar, con

\section{Bibliografía}

1. Johnson LW, Sehon JK, Lee WC, Zibari GB, Mc Donald JC. MIrizzi's syndrome: experience from a multi-institutional review. Am Surg 2001; 67: 11-4.

2. Kok KYY, Goh PYM, Ngoi SS. Management of Mirizzi's syndrome in the laparoscopic era. Surg Endosc 1998; 12: 1242-4.

3. Abou-Saif A, Al-Kawas FH FH. Complications of gallstone disease: Mirizzi syndrome, cholecystocholedocal fistula and gallstone ileus. Am J Gastroenterol 2002; 97: 249-54.

4. Targarona EM, Andrade E, Balagué C, Ardid J, Trías M. Mirizzi's syndrome diagnostic and therapeutic controversies in the laparoscopic era. Surg Endosc 1997; 11: 842-5.

5. Yeh CN, Jan YY, Chen MF. Laparoscopic management for Mirizzi syndrome. Surg Endosc 2003; 17: 1573-8.

6. Cortés Ruiz M, Vázquez García A. Frecuencia del síndrome de Mirizzi en un hospital de enseñanza. Cir Gen 2003; 25: 334-7.

7. Castellón CJ, Fernández M, Del Amo E. Coledocolitiasis: indicaciones de colangiopancreatografía retrógrada endoscópica y colangiorresonancia magnética. Cir Esp 2002; 71: 314-8.

8. Griniatsos J, Wan A, Ghali S, Bentley M, Isla AM. Exploración laparoscópica de la vía biliar. Experiencia de una unidad especializada. Cir Esp 2002; 71: 292-5.

9. England RE, Martin DF. Endoscopic management of Mirizzi's syndrome. Gut 1997; 40: 272-6.

10. Robles Palomar PJ, Lancaster Jones B, García Lara J. Síndrome de Mirizzi, abordaje abdominal laparoscópico. Rev Mex Cir Endoscop 2005; 6: 55-8.

11. Schäfer M, Schneiter R, Krähenbühl L. Incidence and management of Mirizzi syndrome during laparoscopic cholecystectomy. Surg Endosc la apertura de fístulas bilio-biliares y bilio-digestivas. Para obtener un correcto diagnóstico de presunción deberán solicitarse pruebas de imagen, inicialmente ecografía y en segundo término otras más avanzadas, siempre que estén disponibles, como la CRM o el TAC helicoidal con colangiograma. La endoscopia digestiva (CPRE) permitirá el drenaje de la vía biliar y, en enfermos deteriorados, una solución completa y a largo plazo. Por último, tras la estabilización del paciente, la cirugía, abierta o laparoscópica, son electivas para la corrección de la litiasis biliar y la curación definitiva.
2003; 17: 1186-90.

12. Bueno Lledó J, Serralta Serra A, Planells Roig M, Rodero Rodero D. Colecistectomía laparoscópica en el paciente anciano. Cir Esp 2002; 72: 205-9.

13. Lujan JA, Parrilla P, Robles R, Marín P, Torralba JA, García Ayllón J. Laparoscopic cholecystectomy vs open cholecystectomy in the treatment of acute cholecystitis. Arch Surg 1998; 133: 173-5.

14. Maíllo CL, Martín E, López J, Jover JM, Martínez J, Margalet I, et al. Efecto del neumoperitoneo en la hemodinámica venosa durante la colecistectomía laparoscópica. Influencia de la edad de los pacientes y del tiempo de cirugía. Med Clin (Barc) 2003; 120: 330-4.

15. Kama NA, Doganay M, Dolapci M, Reis E, Atli M, Kologlu M. Risk factors resulting in conversion of laparoscopic cholecystectomy to open surgery. Surg Endosc 2001; 15: 965-8.

16. Paganini AM, Incidence and management of Mirizzi syndrome during laparoscopic cholecystectomy. Surg Endosc 2003; 17: 1191-2.

17. Rohatgi A, Singh K. Mirizzi syndrome: laparoscopic management by subtotal cholecystectomy. Surg Endosc 2006; 20: 1477-81.

18. Turégano Fuentes F, Mercader Cidoncha E, Pérez Díaz D, Sanz Sánchez M, Jiménez-Gómez LM. Utilización del ligamento redondo en la reparación de defectos amplios de la vía biliar en el síndrome de Mirizzi de tipo II. Cir Esp 2006; 79: 379-81.

19. García Marcilla JA, Vázquez Rojas JL, Pérez Vicente F, Luri P, Diego Esteve M, Calpena R, Medrano J. Colecistectomía electiva frente a urgente en el paciente anciano, Cir Esp 1998; 63: 365-7.

20. Hazzan D, Golijanin D, Reissman P, Adler SN, Shiloni E. Combined endoscopic and surgical management of Mirizzi syndrome. Surg Endosc 1999; 13: 618-20. 